\title{
Packet Length Adaptation for Energy-Proportional Routing in Clustered Sensor Networks
}

\author{
Chao-Lieh Chen ${ }^{1}$, Chia-Yu Yu ${ }^{2}$, Chien-Chung $\mathrm{Su}^{2}$, \\ Mong-Fong Horng ${ }^{3}$, and Yau-Hwang Kuo ${ }^{2}$ \\ ${ }^{1}$ Department of Electronics Engineering \\ Kun-Shan University, Yung-Kang, Tainan County, Taiwan, R.O.C. \\ frederic@ieee.org \\ ${ }^{2}$ Department of Computer Science and Information Engineering \\ National Cheng Kung University, Tainan, Taiwan, R.O.C. \\ \{kuoyh, yujy, sujz\}@cad.csie.ncku.edu.tw \\ ${ }^{3}$ Department of Computer Science and Information Engineering, \\ Shu-Te University, Kao-Hsiung, Taiwan, R.O.C. \\ mfhorng@mail.stu.edu.tw
}

\begin{abstract}
We study the maximization of throughput and energy utilization in noisy wireless channels by adjusting packet length adaptively to network instant statistics. The optimal packet length adaptation (PLA) for throughput and energy utilization in wireless networks with and without re-transmission is respectively derived and developed. As more noises introducing more energy consumptions, the noises are equivalently regarded as lengthening of transmission distances. Therefore, an equivalent distance model of noisy channels is developed for more accurate estimation of the dissipated proportion in the residual energy such that further improvement of energy utilization and throughput is obtained. We integrate the PLA with the energy-proportional routing (EPR) algorithm for best balance of energy load. Therefore, performance metrics such as lifetime extension, throughput, and energy utilization are maximized even the distribution of channel noise is so un-predictable. Since the equivalent distance is dynamic, we believe that it is useful for network topology re-organization and will be useful in the future work of mobile ad-hoc networks.
\end{abstract}

\section{Introduction}

Wireless channels' inherently error-prone and their time-varying characteristics make it difficult to consistently obtain good performance. Due to limited bandwidth and limited energy, many researches focus on how to increase throughput and utilize energy effectively. However, many of the researches, such as [1-6], do not consider channel noises' accomplice of throughput reduction and energy wasting. In real world's wireless networks, significant packet losses are due to channel noises. In this paper, we study how to effectively increase throughput and energy utilization in noisy 
wireless sensor networks by adjusting packet lengths adaptively to the network statistics. We assume that each packet consists data and header portions. The length of the header in a packet is constant for specific protocols while the length of the data is variable. In addition, the header is an overhead in the wireless networks and we must decrease its relative energy dissipation and at the same time increase throughput of network. To do so, we develop the adaptation method of the data length of packets according to different network instant condition, that is, the bit error rate (BER). As higher noises, which also mean higher BER, introducing more energy consumptions, the noises are equivalently regarded as lengthening of transmission distances. We derive the equivalent distance for a noisy channel and use it for more accurate estimation of energy consumption.

With the adaptation mechanism, it is attractive to apply it in sensor networks. Cooperated with adequate energy-aware routing method, the adaptation mechanism will exploit its advantages of energy utilization and throughput maximization through an extended lifetime of the sensor network. Therefore, we propose the cooperation with energy-proportional routing (EPR) algorithm for noisy clustering-based sensor networks. The EPR was originally proposed that proportionally balances energy loads of nodes and clusters when data amounts and packet lengths of wireless links vary $[11,12]$. For inter-cluster communications, the EPR makes all clusters dissipate the same proportion of its remaining energy when transmitting data to the base station. Even though the clusters have quite different data amounts and distances to the base station, with EPR, data loads are shared evenly and no cluster nodes die early due to having been busy transmitting data for a long time. For intra-cluster communication, the EPR also successfully balances the energy load. Due the energy-proportional balance, both the throughput is increased and lifetime is extended. Many energy aware routing schemes [1-6] use constant packet length for transmissions and assume constant data amount each round. The EPR works well no matter how the data amount and the packet length vary with time. Therefore, the cooperation of the PLA and EPR effectively provides very good energy and throughput performances even if the noises in the network are dynamic and so unpredictable. Some other load-balance routing protocols for clustering-based sensor networks can be found in [2-6]. However, the proposed EPR routing algorithm outperforms in balancing the loads especially in the noisy environments.

The rest of the paper is organized as follows. Section 2 introduces the radio energy model in wireless networks and describes the noise models, which are used in the wireless sensor networks. With the noise models we can formulate the problem suffered by wireless communication ignoring noise effects. Section 3 provides the analyses of throughput, energy utilization and equivalent distance for noisy channels. With the analyses, we derive the optimal packet length for the PLA algorithm. Section 4 introduces the EPR routing with the PLA for clustering-based wireless sensor networks. In Section 5, we present the simulations and compare the performance of the proposed adaptation to the ones of using constant packet lengths and adopting error-free routing protocols. In Section 6, we conclude our idea and propose future works. 


\section{Problem Formulation}

The energy dissipated for data transmission and receiving in the air has direct effect on the lifetime in wireless sensor networks. To utilize energy consumption, there has been a significant amount of research in the area of low-energy radios. In this paper we adopt the widely accepted energy model $[1-6,11,12]$ for energy consumption estimation. The energy model was proposed where the transmitter dissipates energy to run the radio electronics and the power amplifier as well as the receiver dissipates energy to run the electronics. The power attenuation is dependent on the distance $d$ between the transmitter and receiver. The propagation loss can be modeled as inversely proportional to $d^{2}$. To transmit $L$-bit message over a distance $d$, the radio expends:

$$
E_{T x}(L, d)=L E_{\text {elec }}+L \varepsilon_{\text {amp }} d^{2}
$$

while for receiving this message, the radio expends

$$
E_{R x}(L)=L E_{\text {elec }}
$$

The electronics energy, $E_{\text {elec }}$ depends on factors such as the digital coding, modulation and filtering of the signal before it is sent to the transmitting amplifier. According to (1), the total energy $E_{T x}(L, d)$ dissipated by a node for data transmission is a function of the topological parameter $d$ and data amount $L$. On the other hand, the energy $E_{R x}(L)$ used for receiving data from other clusters or nodes accounts for only data amount $L$. The dissipated energy is related to packet length and the distance between transmitter and receiver. In addition, when we consider transmission in noisy networks, the packet error rate (PER) is related to packet length. For a given BER, the longer the packet length is, the larger packet error rate is. In contrast to noiseless cases, the throughput will be worse adopting longer packet length for transmission.

When we transmit packets over noiseless channel, no packets will be lost in this channel. Therefore, energy is not wasted in transmitting incorrect packets. However, when transmitting packets over noisy channel, packets are influenced by noise and corrupted packets are dropped if error correction mechanism such as ECC cannot recover the error bits. When packets are dropped, energy is wasted. Therefore, if packet length is too long in noisy wireless networks, this will result in large PER and waste much energy in lost packets. Although short packet length causes smaller PER, the transmission energy used by packet header is relatively larger. On the other hand, the throughput is also downgraded if the data proportion is small in a packet. In this paper, we derive the optimal packet length with respect to wireless network noise condition. The optimal packet length will result in larger throughput and utilizing energy effectively.

To reveal the effect of the packet length to throughput and energy consumption, we consider the noise model as follows. The common noisy models in wireless networks are divided into Gaussian channel [13], Ricean channel [9], and Rayleigh channel [8]. Since Gaussian channel is the most commonly used model in noisy wireless networks, we primarily consider the Gaussian channel in this paper. We express PER (packet error rate) in terms of the packet length $L$ and bit error rate in a packet. Let $P(m, L)$ be 
defined as the probability that a packet of length $L$ bits contains $m$ erroneous bits. For a general channel, if MAC FEC is employed at the data link layer, we assume that $t$ channel bits errors will be corrected. Therefore, we can get the following formula:

$$
P E R=\sum_{m=t+1}^{L} P(m, L)=1-\sum_{m=0}^{t} P(m, L)
$$

If MAC FEC is not employed (i.e., in (3), $t=0$ ), we can reduce PER to the following formula:

$$
P E R=1-P(0, L)
$$

Since Gaussian Channel can be converted to binary symmetric channel [13], we have the following expression for PER in terms of packet length and BER $p_{b}$ :

$$
P E R=P_{e}=\sum_{i=1}^{L} p_{b}^{i}\left(1-p_{b}\right)^{L-i}=1-\left(1-p_{b}\right)^{L} \approx L p_{b} .
$$

The PER represents that packets will be dropped whenever any one bit is incorrect in this packet. As we can see from (5), the PER is about proportional to the packet length $L$. When packet drops, throughput and energy utilization degrade. In Section 3, we will derive the optimal packet length in terms the bit error rate and header length when using Gaussian channel model so that we can adapt the packet length to optimally improve throughput and energy utilization.

\section{Optimal PLA and Equivalent Distance}

First, we consider that the packet must be retransmitted after the packet is lost. The probability that packet must be transmitted $n$ times is $\left(1-p_{e}\right) p_{e}^{N-1}$. The expected number of required transmissions until the packet is received correctly is:

$$
\bar{N}=\sum_{n=1}^{N} n\left(1-p_{e}\right) p_{e}^{n-1}=\frac{1}{1-p_{e}} \approx\left(1-p_{b}\right)^{-L}
$$

We approximate the throughput and energy dissipation in terms of this expectation number.

\subsection{Throughput}

In case that retransmission is required, from (6), the average of required transmission time for every packet is as follows.

$$
\bar{T}=\bar{N} \frac{L}{R}=\frac{L\left(1-p_{b}\right)^{-L}}{R}
$$


where $R$ is the bandwidth. Assume that the header length $h$ is constant and the data length is variable. Therefore, the respective throughput with and without retransmission are

$$
\begin{gathered}
T h=\frac{L-h}{\bar{T}}=\frac{(L-h)}{L} R\left(1-p_{b}\right)^{L} \\
T h=\frac{(L-h)}{L} R\left(1-L p_{b}\right)
\end{gathered}
$$

For sufficiently long packet, equations (8) and (9) are close.

\subsection{Energy Utilization}

In this paper, the distance of a sensor node pair is constant since sensors have fixed position after they are deployed. The energy consumed per bit data transmission between any two nodes is regarded as a constant $E$. We use the metric of energy utilization to decide the optimal packet length. The energy utilization is defined as the total useful data sent by unit amount of energy [7]. According to this definition, to obtain the energy utilization, we need to calculate the ratio of the effective data amount transmitted to the total energy consumed by a node for this transmission. We also consider the energy utilization in two cases - requirement of retransmission or not. The respective expected energy utilizations with and without retransmission are

$$
\begin{gathered}
E_{u t}=\frac{L-h}{L \bar{N} E}=\frac{(L-h)\left(1-p_{b}\right)^{L}}{L E} \\
E_{u t}=\frac{L-h}{L \bar{N} E}=\frac{(L-h)\left(1-L p_{b}\right)}{L E}
\end{gathered}
$$

Summarizing (8) to (11), the optimal packet length maximizing the throughput and best utilizing energy is the one making the derivative of (10) or (11) zero. The respective optimal packet lengths with and without retransmission are

$$
\begin{gathered}
L_{o p t}=\frac{h}{2}+\sqrt{\frac{h^{2}}{4}-\frac{h}{\ln \left(1-p_{b}\right)}} \\
L_{o p t}=\sqrt{\frac{h}{p_{b}}} .
\end{gathered}
$$

\subsection{Equivalent Distance}

As more noises introducing more energy consumptions, the noises are equivalently regarded as lengthening of transmission distances. Because lost packets will waste 
energy and energy is the function of communication distance, we assume that the wasted energy is added to the communication distance such that packets are received correctly as in perfect channel. Therefore, the equivalent distance model of a noisy channel is derived for more accurate PLA such that further improvement of energy utilization and throughput is performed. Because bit error rate is time-variant, the equivalent distance will also be time-variant. When perform energy-aware routing such as EPR, the equivalent distance will be very useful in balancing the energy load.

The relationship between energy and equivalent distance is as follows (we only consider that retransmission of lost packets will not required). From section 2, we know that the energy model is as equation (1) and in this section we also have energy utilization expressed as (11) Combining (1) and (11), we can get the following equation.

$$
E_{u t}=\frac{(L-h)\left(1-L p_{b}\right)}{L E_{\text {elec }}+L \varepsilon_{\text {amp }} d^{2}}
$$

Let the equivalent distance be $d_{e q}$ in perfect channel. Therefore, we can get the following equation:

$$
\frac{L-h}{L E_{\text {elec }}+L \varepsilon_{\text {amp }} d_{e q}{ }^{2}}=\frac{(L-h)\left(1-L p_{b}\right)}{L E_{\text {elec }}+L \varepsilon_{\text {amp }} d^{2}}
$$

Finally, we the equivalent distance is derived as follows.

$$
d_{e q}=\sqrt{\frac{\varepsilon_{a m p} d^{2}+L p_{b} E_{e l e c}}{\varepsilon_{a m p}-L p_{b} \varepsilon_{a m p}}}
$$

In Section 4, we perform EPR routing by using the equivalent topology constituted by the equivalent distances among sensor nodes.

\section{Energy-Proportional Routing with Optimal PLA}

In each round, as shown in Fig. 1, each sensor node statistically calculates the packet error rate $p_{e}$ by number of its transmitted packets and the received packets. This statistic can be done in several ways. In this paper we assume that the destination sensor acknowledges the total number of received packets to its source at the end of a round. Dividing the accumulated number of successfully sent packets by total number of sent packets, we have the PER. In the setup phase of the coming round, with the PER, each sensor node inversely calculates the BER $p_{b}$ according to (5) (lines 2-3 in Fig. 1) and then calculates the optimal packet length according to equations (12) to (13)(line 5 in Fig. 1). Consequently, each sensor node will transmit data by using the optimal packet length and the equivalent distances (Eq. (16) and line 6) in the communication phase.

In $[11,12]$, an effective energy-proportional routing (EPR) algorithm for lifetime extension was proposed. In the EPR algorithm, to optimally utilize energy, light-load 
sensor nodes that conserve energy are ideal candidates as intermediate nodes for forwarding data from others. To balance the load, first, the EPR algorithm predicts energy consumption of each node in each round. Then the algorithm controls the energy consumption of each node (cluster) as close as possible to the mean value of energy utilizations among nodes (clusters). Finally, the algorithm checks satisfaction of the energy constraints in terms of distances and predicted data amounts. The algorithm performs routing by determining whether a node or a cluster head should either undertake forwarding tasks or transmit data to intermediate hops. In this way, energy dissipation is evenly distributed and the lifetime of the whole wireless sensor network is ultimately extended.

When a source node transmits the gathered data to the base station via intermediate nodes, the intermediate nodes can evenly share the responsibility of the load in a round. The concept is called energy-proportional balance. The EPR effectively extends lifetime and increases throughput in both intra- and inter-cluster communications. Unlike many state-of-the-art routing schemes [1-6] that assume constant data amount and packet length in each round, the EPR works well no matter how dynamics of the data amount and packet length are. Therefore, considering integration of the packet PLA with routing schemes, the EPR is the best candidate. In this paper, we perform the optimal PLA for both intra- and inter-cluster communications that use EPR. For inter-cluster communications, the operation steps of the EPR algorithm in the setup phase are as follows. First, sensor nodes are formed into clusters by replacing geometrical distances with the equivalent distances in conventional algorithms such as those in many state-of-art clustering algorithms for sensor networks. Second, every

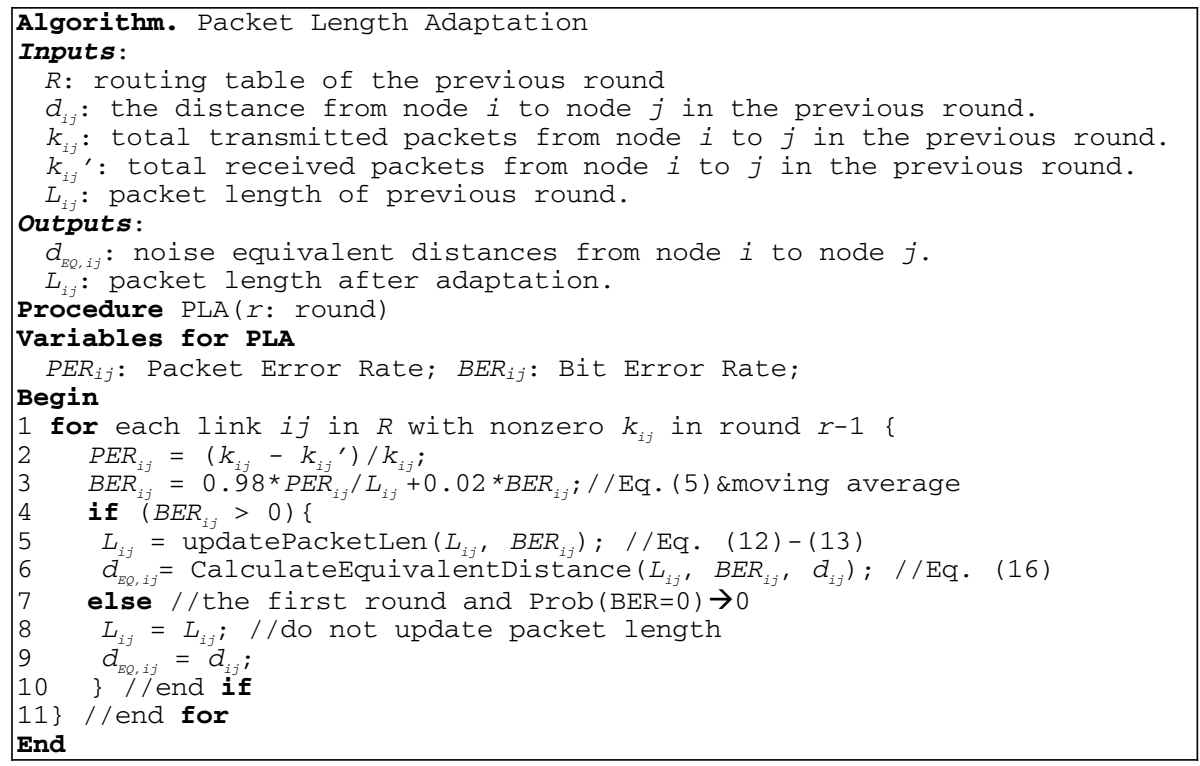

Fig. 1. The pseudo code of the PLA 
cluster head predicts the amount of data to be transmitted according to the Markov model. The prediction and the PER statistics are useful in calculating local energy utilization for each cluster head. Finally, for those cluster heads having higher energy dissipation than the energy mean value $\omega_{t h}$, we adopt EPR routing algorithm to direct their data to those clusters having been less active for an extended period and having much more remaining energy. We refer the readers to [11, 12] for more details about the EPR. The packet transmission in the communication phase uses the optimal packet length calculated by (12) and (13) in the setup phase. In this way, while the total energy dissipation of the sensor network is kept evenly distributed, simultaneously the noise impacts on energy wasting and throughput reduction are eased.

\section{Experiments and Simulations}

In this section, we simulate the proposed model by using NS2 network simulator. We compare the performance with other protocols by using some factors including collected data, throughput and energy utilization in the base station. For these experiments, we randomly distribute 100 nodes in an area $100 \times 100 \mathrm{~m}^{2}$. The base station is located at location $(X=0, Y=0)$. The bandwidth of the channel was set to 1 Mbps. The radio electronic energy was set to $50 \mathrm{~nJ} / \mathrm{bit}$ and the radio transmission energy was set to $10 \mathrm{pJ} / \mathrm{bit} / \mathrm{m}^{2}$. These parameters are the same as in the $\mathrm{LEACH}$ protocol [1].

\subsection{The Effects of Noise}

When packet length is 500 bytes, the distribution of the number of erroneous bits in packets is obtained by statistical accumulation in the NS2 simulator. Fig. 2(a) and Fig. 2(b) respectively show the effect on throughput and energy utilization under different bit error rates. These two figures also validate equations (8)-(11). From Fig. 2(a) and Fig. 2(b), we need to use different optimal packet lengths under different BER status.
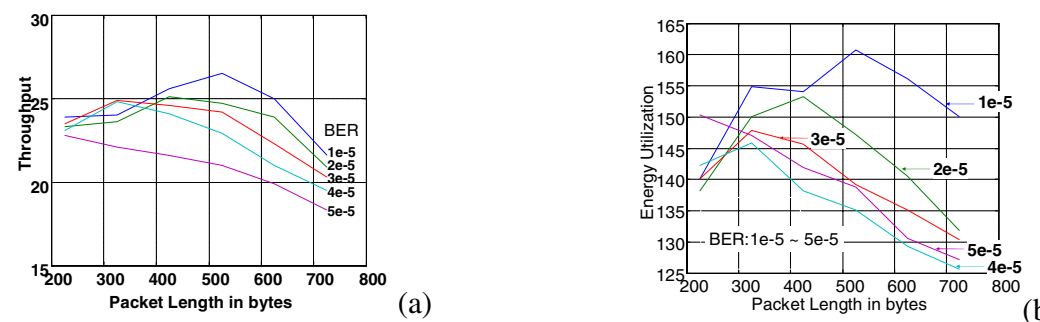

Fig. 2. The effect of BER: (a) the throughput of network under different bit error rates, and (b) the energy utilization under different bit error rates 


\subsection{Simulations of the Whole Sensor Network}

The initial packet length for each link is 500 bytes and after that the PLA algorithm adapts the packet length. The header length of packets is 25 bytes and lost packets are not retransmitted. We present simulation results of total data received by the base station in lifetime, throughput, and energy utilization. The results of these three metrics are respectively shown in Fig. 3(a), (b), and (c). From the results, we know that adjusting the packet length dynamically by PLA in noisy wireless sensor network increases throughput and energy utilization. Moreover, cooperating with EPR, we gain further benefits. The legends of the results are "Proposed" -- the proposed EPR routing with PLA, "LEACH(Adaptive Length)" -- the LEACH-c routing with PLA, "LEACH(Length=500)" -- the LEACH-c algorithm with constant packet length 500 bytes, "LEACH(No Error)" -- original LEACH-c algorithm with constant packet length 500 bytes and zero BER, and "Length=aaa, EPR" -- EPR routing with constant packet length aaa $\in\{300,400,500,600,700\}$.
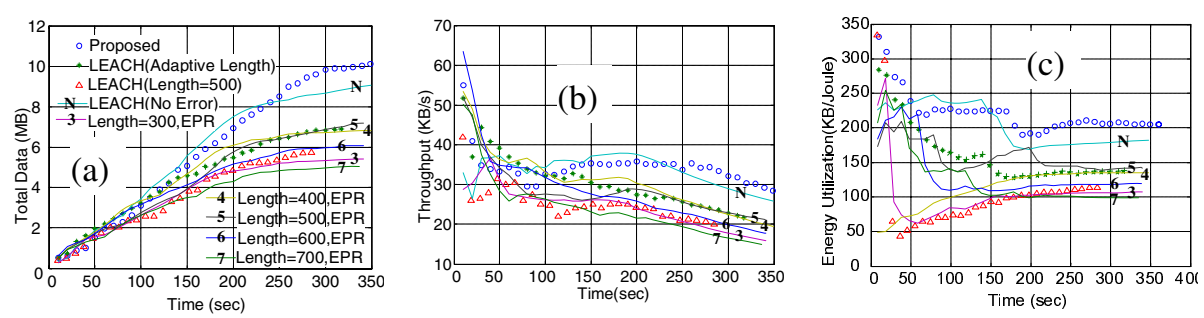

Fig. 3. Simulation results of the whole sensor network: (a) Total data received by base station, (b) the throughput of network, and (c) the energy utilization of network.

\subsection{Equivalent Distance}

Communication distance is an important factor in estimating energy consumption in wireless networks. In the previous subsection, when calculating the dissipated energy proportion in the residual energy, the EPR uses real geometrical distance in the energy model. Here we adopt the noise equivalent distances rather geometrical ones to more accurately estimate the energy proportion caused by noises including geographical issues such as obstacles. With BER and packet length obtained in PLA, we use equation (16) to compute the equivalent distance. With the equivalent distances, clusters are organized and routing table is generated in each round. The comparisons of using and not using the equivalent distance are as Fig. 4. The performance of EPR with PLA is further improved if using equivalent distance in energy consumption estimation. We compare the proposed method with other protocols, such as PEGASIS [2, 3], BCDCP [6], and LEACH-c [1] to see how the noise impacts network performances. We use the normalized performance ratio to show the comparison results. The following normalized performance ratios are acquired by comparing with $\mathrm{LEACH}$ protocol. According to 

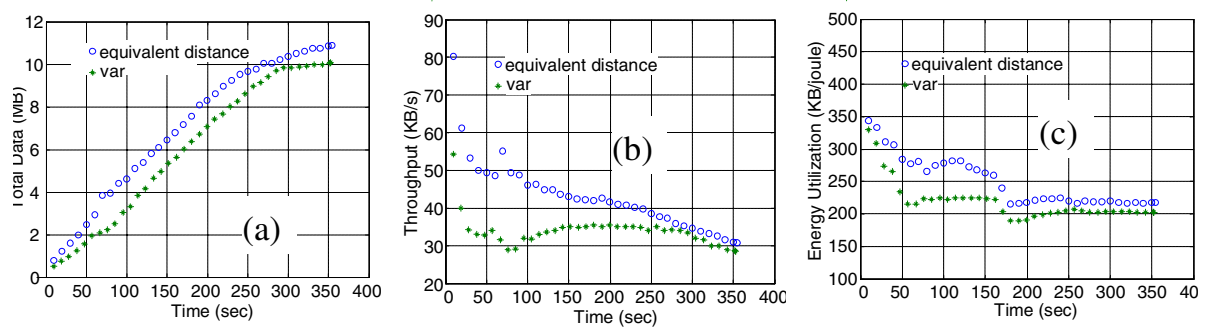

Fig. 4. The simulation results for equivalent distance: (a) total data received by the base station, (b) throughput, and (c) the energy utilization of the sensor network. Legend "var" represents EPR with PLA but without equivalent distance for energy consumption estimation.

[10], using perfect channels, the normalized performance ratio of PEGASIS [2, 3], BCDCP [6], and LEACH-c are 1.5, 1.67, and 1.167 respectively. From Fig. 4, the performance of the proposed method is 1.76 even under noisy environment. These ratios reveal that the channel noise obviously affects the energy balance.

\section{Conclusion and Future Works}

In this paper, we propose the packet length adaptation (PLA) scheme according to the derivation of optimal packet length in the noisy channels. The packet length adaptation successfully increases throughput and energy utilization of clustered sensor networks. Among state-of-the-art routing schemes for sensor networks, the energy-proportional routing $(\mathrm{EPR})$ is superior especially when the packet length and the data amount are randomly distributed in a round. Therefore, we gain further improvement in throughput and energy utilization when the packet length adaptation is cooperated with the energy-proportional routing. With the optimal packet length for each instant network situation, the noise-equivalent distance is obtained and is useful for estimation of dissipated energy proportion in the residual energy. Therefore, the EPR routing optimally balance the energy load among sensor nodes and clusters. As BER is time-variant, the equivalent distance is also time-variant. In the future, we will apply the proposed method in mobile ad-hoc networks where distances among nodes are time-variant. Furthermore, mathematical analyses based on various noise models and different MAC sub-layers will be continued.

\section{References}

1. W. Heinzelman, A. Chandrakasan, and H. Balakrishnan, "An Application-Specific Protocol Architecture for Wireless Microsensor Networks", IEEE Transactions on Wireless Communications, 1(4) (2002) 660-670.

2. S. Lindsey, C. Raghavendra, and K. M. Sivalingam, "Data Gathering Algorithms in Sensor Networks Using Energy Metrics," IEEE Transactions on Parallel and Distributed Systems, 13(9) (2002) 924-935. 
3. S. Lindsey, C. Raghavendra, "PEGASIS: Power-Efficient Gathering in Sensor Information Systems," IEEE Aerospace Conference Proceedings, 3 (2002) 1125-1130.

4. O. Younis and S. Fahmy, "Distributed Clustering in Ad-hoc Sensor Networks: A Hybrid, Energy-Efficient Approach," IEEE Transactions on Mobile Computing, 3(4) (2004) 366-379.

5. O. Younis and S. Fahmy, "Distributed Clustering in Ad-Hoc Sensor Networks: A Hybrid, Energy-Efficient Approach," Proceedings of IEEE INFOCOM, (2004).

6. S. D. Muruganathan, D. C. F. MA, R. I. Bhasin, and A. O. Fapojuwo, "A Centralized Energy-Efficient Routing Protocol for Wireless Sensor Networks," IEEE Radio Communications, 43(3) (2005) S8-S13.

7. X. Wang, J. Yin and D. P. Agrawal, "Effects of Contention Window and Packet Size on the Energy Efficiency of Wireless Local Area Network", Proceedings of 2005 IEEE Wireless Communications and Networking Conference, 1 (2005) 94 - 99.

8. H. Bischl and E. Lutz, "Packet error rate in the non-interleaved Rayleigh channel," IEEE Transactions on Communications, 43 (1995) 1375-1382.

9. Ruggero Reggiannini, "A lower performance bound for phase estimation over slowly-fading Ricean channels", Global Telecommunications Conference, 3 (1995) 2012-2016.

10. Siva D. Muruganathan, Daniel C. F. Ma, Rolly I. Bhasin and Abraham O. Fapojuwo, "A Centralized Energy-Efficient Routing Protocol for Wireless Sensor Networks", IEEE Radio Communications, (2005) S8-13.

11. Chao-Lieh Chen and Kuan-Rong Lee, et al., "An Energy-proportional Routing Algorithm for Lifetime Extension of Clustering-based Wireless Sensor Networks," Workshop on Wireless, Ad Hoc, and Sensor Networks, http://acnlab.csie.ncu.edu.tw/WASN, (2005), Taiwan.

12. Chao-Lieh Chen and Kuan-Rong Lee, et al., "An Energy-proportional Routing Algorithm for Lifetime Extension of Clustering-based Wireless Sensor Networks", Journal of Pervasive Computing and Communications, to appear in 2 (2006).

13. Thomas M. Cover and Joy A. Thomas, Elements of Information Theory, 1991. 